\title{
Lutein concentration in human milk during early lactation and its relationship with dietary lutein intake
}

\author{
Hellas Cena ${ }^{1, *}$, Anna Maria Castellazzi ${ }^{2}$, Amedeo Pietri ${ }^{3}$, Carla Roggi ${ }^{1}$ and \\ Giovanna Turconi ${ }^{1}$ \\ 'Department of Applied Health Sciences, Section of Human Nutrition and Dietetics, Faculty of Medicine, \\ University of Pavia, Via Bassi 21, I-27100 Pavia, Italy: ${ }^{2}$ Department of Paediatric Sciences, Faculty of Medicine, \\ University of Pavia, Pavia, Italy: ${ }^{3}$ Institute of Food Science and Nutrition, Agricultural Science Faculty, Catholic \\ University of the Sacred Heart of Piacenza, Piacenza, Italy
}

Submitted 30 January 2008: Accepted 7 December 2008: First published online 16 February 2009

\begin{abstract}
Objective: The present study aimed to estimate the lutein concentration in human milk during early lactation and its relationship with dietary lutein intake measured through the administration of a short FFQ.

Design: A cross-sectional study in which an FFQ was administered twice: on day 3 (T0) and day 30 (T1) postpartum; meanwhile two breast milk samples were collected. Maternal plasma samples were obtained at T0. The comparison of dietary lutein intakes and likewise lutein concentrations in breast milk at T0 and T1 were analysed with Student's $t$ test. Pearson's correlation coefficient was used to determine the association between dietary lutein intake and lutein concentration in milk and plasma, respectively, as well as the correlation between breast milk and plasma lutein concentrations at TO.

Setting: Pavia, northern Italy.

Subjects: Twenty-one pregnant women, age range 24-42 years, were recruited during their last trimester on a voluntary basis.

Results: Both breast milk and plasma lutein concentrations were significantly correlated with dietary lutein intake $(r=0.86, \quad P=0.0001$ and $r=0.94$, $P=0 \cdot 0001$, respectively). There was a clear significant correlation between milk and plasma lutein concentrations $(r=0 \cdot 87, P=0 \cdot 0001)$. Mature milk lutein concentration, although significantly reduced at T1 $(P<0 \cdot 01)$, maintained a fairly high correlation with dietary lutein intake $(r=0 \cdot 82, P=0 \cdot 0001)$.

Conclusions: Even though milk lutein concentration decreased during early lactation, it remained significantly correlated with daily lutein intake. Therefore, while awaiting further research, dietary recommendations advising intake of fresh fruit and vegetables rich in lutein, throughout the whole duration of pregnancy and lactation, are extremely useful.
\end{abstract}

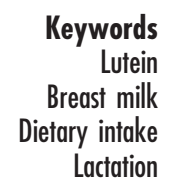

Lutein is a xanthophyll carotenoid found particularly in dark-green leafy vegetables. It is widely distributed in tissues and is one of the principal carotenoids in the eye lens and macular region of the retina, being dominant in the more external portions of the retina ${ }^{(1,2)}$. Owing to its absorbent properties in relation to luminous radiation and antioxidant capabilities, this xanthophyll is an interesting compound for the health of the eye $\mathrm{e}^{(3-5)}$. The action of lutein appears to be important in this context thanks to its considerable filtering capacity as regards light ${ }^{(6)}$ and its antioxidant action.

There are still few studies that have highlighted the possible role of lutein in the long-term prevention of morbidity and disability in neonates. The oxidative stress to which an infant is subjected at birth has been considered a risk factor in the aetiology of damage to the visual apparatus and more besides. As for age-related macular degeneration in the elderly, where free radicals are today considered an important risk factor, even in neonates, antioxidants could play a protective role. Lutein, especially, may have an action countering the pro-oxidant effects of light by absorbing radiation and neutralizing singlet oxygen and free radicals that are produced in the metabolic processes of retinal ischaemic tissue ${ }^{(7)}$.

Among its components, breast milk has a large amount of antioxidants, including lutein, that may play an essential role for newborn eye health, given that this carotenoid seems to have specific transporters widely represented at the level of the placenta, the mammary gland and the ocular tissue ${ }^{(8)}$. This carotenoid may protect 
the neonate's retina from light-induced oxidative damage that it may encounter in the first months of life ${ }^{(9,10)}$; indeed, in this period, lutein in breast milk may help to accommodate the newborn's transition from the darkness experienced in utero.

Levels of lutein in human milk are two to three times higher than those of $\beta$-carotene ${ }^{(10)}$; thus maternal milk is the main dietary source of lutein for infants until weaning occurs. Investigation of maternal milk lutein concentration during the first month postpartum may provide information useful to suggest dietary recommendations during both pregnancy and lactation, in order to satisfy both infant and maternal requirements. The few studies that have been conduced in lactating women report great variability of dietary lutein intakes among countries, ranging from 2108 to $4929 \mu \mathrm{g} / \mathrm{d}^{(11,12)}$.

The present study aimed to estimate the lutein concentration in human milk during early lactation in a group of puerperae and its relationship with dietary lutein intake measured through the administration of a short $\mathrm{FFQ}^{(13)}$.

\section{Materials and methods}

\section{Sample}

Twenty-one pregnant women were recruited on a voluntary basis, during their last trimester, at the Obstetric Unit of Pavia University in northern Italy.

Written informed consent was obtained from all participants prior to their inclusion in the study, which was performed in accordance with the ethical standards laid down in the appropriate version of the 1994 Declaration of Helsinki and approved by the University of Pavia's Faculty of Medicine Ethical Committee.

Subjects, in good health and nutritional state as assessed by anthropometric measurements and laboratory tests, were eligible to join the study if they did not smoke, had not taken prenatal supplements containing lutein, did not consume alcohol and had no unusual dietary habits due to spontaneous intake restrictions, vegetarianism, known food allergies, etc.

Six women dropped out during the trial period.

\section{Study design}

In the present cross-sectional study, a previously validated $\mathrm{FFQ}^{(13)}$ was administered twice: on day 3 (T0) and day 30 (T1) postpartum, in order to estimate dietary lutein intake; meanwhile, two breast milk samples were collected. In addition, maternal plasma samples were obtained on the third day of puerperium (TO).

\section{Intake assessment}

A brief FFQ was previously developed ${ }^{(13)}$ providing a list of fruit and vegetables typically consumed by the Italian adult population according both to the Mediterranean diet $^{(14)}$ and the FFQ used in the European Prospective Investigation into Cancer and Nutrition ${ }^{(15,16)}$, ending up with a final list of thirty items (dark-green leafy vegetables as well as green peas, summer squash, broccoli, lettuce and corn; fruits such as tangerines, peaches and oranges). The FFQ was then validated by comparing the results obtained with a $7 \mathrm{~d}$ dietary record as a reference standard ${ }^{(13)}$.

\section{Data collection}

We chose to administer the questionnaire by interview since some problems may arise with self-administration; for example, answers may be incomplete as some respondents will only complete the questionnaire for items they usually eat.

Frequency of usual food consumption was investigated by inviting the respondents to report their consumption as 'never consumed' or in units of their choice such as 'number of occasions' per day, week or month, rather than being restricted to specific frequency ranges with a reference period of 'over the past month' as already reported by other authors ${ }^{(17,18)}$. Usual dietary lutein intake was assessed with this quantitative thirty-item FFQ.

Summary questions aimed at obtaining overall information on the number of servings of fruit and vegetables per day, as well as questions for cross-checking the consistency of answers, were asked during the interviews.

To quantify the food portions sizes consumed, a photographic food atlas was used ${ }^{(19,20)}$.

Patients reported that the FFQ was easy to understand, clear, short and not demanding. Furthermore, the use of the colour photographic food atlas helped to hold the attention of the subjects being interviewed.

All interviews were performed at the Obstetric Unit of Pavia University by a highly trained dietitian who had received $3 \mathrm{~h}$ of instruction. Completing the questionnaire took on average $10 \mathrm{~min}$. The questionnaire was administered twice, when the milk samples were being taken.

\section{Collection of milk and blood samples}

The breast milk and blood samples were collected in the period immediately following delivery: on day 3 postpartum (T0). Fifteen subjects provided a second milk sample on day 30 (T1) postpartum.

The blood samples were taken after an overnight fast from the ulnar vein, in a quantity of $10 \mathrm{ml}$. The samples were transferred to test tubes containing $0 \cdot 1 \%$ EDTA, subsequently conserved in ice at $4^{\circ} \mathrm{C}$ and transported on dry ice to the analysis laboratory within $4 \mathrm{~h}$. The blood was then centrifuged for $20 \mathrm{~min}$ at $4^{\circ} \mathrm{C}$ and $3000 \mathrm{~g}$ in order to separate the plasma. Two millilitres of plasma from each sample were then analysed.

Milk samples were taken using the breast pump available at the university obstetric clinic. The milk was obtained from one breast in an amount equal to about 5-6 ml. After collection, the milk samples were protected from light sources and also transported on dry ice directly to the laboratory, 
where they were quantified and conserved at $-80^{\circ} \mathrm{C}$. The analyses of these samples were performed within a month of collection, a period during which the analytes remain stable according to the literature ${ }^{(21)}$.

\section{Lutein analysis in milk and plasma samples}

All analyses were conducted under subdued lighting to avoid degradation of lutein. Samples were allowed to thaw and come to room temperature on a shaker.

Lutein (standard) and $\beta$-apo- 8 -carotenal (internal standard) were obtained from Kemin Industries and Fluka, respectively.

Milk ( $2 \mathrm{ml}$ ) was saponified by adding $0.5 \mathrm{ml}$ of potassium hydroxide ( $40 \%$ in methanol) and $0 \cdot 1 \mathrm{ml}$ of $\beta$-apo$8^{\prime}$-carotenal (internal standard, $20 \mu \mathrm{mol} / \mathrm{l}$ ) in methanol. The sample was placed in a water bath at $45^{\circ} \mathrm{C}$ for $30 \mathrm{~min}$ and then lutein was extracted from the saponified matrix using $1.5 \mathrm{ml}$ of hexane (containing $0.01 \%$ butylated hydroxytoluene $(\mathrm{BHT}) \mathrm{w} / \mathrm{v})$ for three times. Combined extracts were dried under nitrogen and then reconstituted in $0.5 \mathrm{ml}$ of isopropanol-hexane $(10: 90 \mathrm{v} / \mathrm{v}) ; 50 \mu \mathrm{l}$ were injected onto the column ${ }^{(22)}$.

Plasma $(0.5 \mathrm{ml})$ was mixed with ethanol $(1 \mathrm{ml})$ containing $\beta$-apo- $8^{\prime}$-carotenal (internal standard, $3 \mu \mathrm{mol} / \mathrm{l}$ ) and BHT $(0 \cdot 01 \% \mathrm{w} / \mathrm{v})$, ethyl acetate $(1 \mathrm{ml})$ and $\mathrm{n}$-hexane $(1 \mathrm{ml})$. The mixture was vortexed and then centrifuged (1 min at $1200 \mathrm{~g}$ ). The supernatant solution was separated, then the pellet was vortexed and centrifuged as before with hexane $(1 \mathrm{ml})$ twice. Water $(0.5 \mathrm{ml})$ was added to the pooled supernatant solution, which was vortexed and centrifuged as before. The hexane phase was carefully pipetted into a glass tube, evaporated to dryness under a stream of nitrogen and then reconstituted in $0.5 \mathrm{ml}$ isopropanol-hexane $(10: 90 \mathrm{v} / \mathrm{v}) ; 50 \mu \mathrm{l}$ were injected onto the column ${ }^{(23)}$.

Samples were analysed with a Thermo Separation Product HPLC, equipped with a P200 pump, an AS3000 autosampler and a Spectra Focus scanning detector, set to $450 \mathrm{~nm}$. A straight phase gradient analysis was carried out, using a Merck Superspher Si 60 column $(4 \mu \mathrm{m}$, $4 \mathrm{~mm} \times 250 \mathrm{~mm}$ ); the mobile phase was a mixture of 2-propanol (A) and hexane (B), with a gradient program as follows:

1. Isocratic $0: 100(\mathrm{~A}: \mathrm{B})$ for $2 \cdot 5 \mathrm{~min}$;

2. Linear gradient from $0: 100$ to $18: 82$ (A:B) from $2 \cdot 5$ to $18 \mathrm{~min}$;

3. Isocratic 18:82 (A:B) from 18 to $24 \mathrm{~min}$;

4. A 2-min gradient back to 0:100 (A:B).

Flow rate was $1 \mathrm{ml} / \mathrm{min}$ and retention time of lutein was about $20 \mathrm{~min}$. In addition to the internal standard, spiking experiments were performed using breast milk, in order to ensure adequate recovery of lutein. The average recovery for five replicate analyses was $85.4 \%$ and the range was $82 \cdot 1-88 \cdot 8 \%$. The limit of detection in milk and plasma was $0 \cdot 025$ and $0 \cdot 100 \mu \mathrm{mol} / 1$, respectively.

\section{Statistical analysis}

The FFQ were analysed and lutein intakes were calculated using the US Department of Agriculture-National Cancer Institute Carotenoids Database ${ }^{(24)}$. Dietary intakes of lutein, estimated on the basis of the FFQ, were analysed by calculating the means and standard deviations.

There were two repeated measures for milk values as well as two repeated estimations for dietary lutein intakes (day 3 and day 30 postpartum), while plasma analyses included only one measure (day 3 postpartum). Comparison between FFQ administered at T0 and T1 on fifteen women to estimate intake of lutein, and likewise comparison of milk lutein concentration (at T0 and T1 on the same sample), was analysed with the paired $t$ test. A normal probability plot was used to assess whether or not the data sets were normally distributed.

Pearson's correlation coefficient was used in order to determine the association between dietary lutein intake and lutein concentration in milk and plasma, respectively. The same coefficient was also used to calculate the correlation between breast milk and plasma lutein concentrations at T0.

Data were analysed with the SPSS statistical software package version 13 (SPSS Inc., Chicago, IL, USA). All results are reported as means and standard deviations.

\section{Results}

Out of twenty-one women enrolled, only fifteen completed the study. Of the six subjects who dropped out, two changed their mind and decided to go elsewhere for their 1-month postpartum check-up, one started smoking and three decided to suspend breast-feeding for formula milk feeding.

Most of the subjects were middle-class and well educated, age range 24-42 years (Table 1). Approximately

Table 1 Characteristics of the sample: lactating women (n 21) aged 24-42 years, Pavia, Italy

\begin{tabular}{|c|c|c|}
\hline & Mean & SD \\
\hline \multirow[t]{2}{*}{ Age (years) } & $33 \cdot 9$ & $4 \cdot 37$ \\
\hline & \multicolumn{2}{|c|}{ No. of subjects } \\
\hline \multicolumn{3}{|l|}{ Education level } \\
\hline Primary school & & \\
\hline Secondary school & & \\
\hline University student & & \\
\hline Graduated & & \\
\hline \multicolumn{3}{|l|}{ Social condition } \\
\hline Teacher/employee/shopkeeper & & \\
\hline Manager & & \\
\hline Unemployed/housewife & & \\
\hline Other & & \\
\hline \multicolumn{3}{|l|}{ Number of deliveries } \\
\hline Nulliparous & & \\
\hline Primiparous & & \\
\hline Multiparous & & \\
\hline
\end{tabular}


half of the subjects had taken prenatal dietary supplements regularly, which however did not contain lutein.

At T0, estimated dietary intake of lutein obtained from the FFQ was 1242 (SD 113) $\mu \mathrm{g} / \mathrm{d}$; breast milk lutein and plasma lutein concentrations in the whole sample ( $n$ 21) were $0 \cdot 26$ (sD 0.19) and 0.69 (sD 0.49) $\mu \mathrm{mol} / 1$, respectively.

Both breast milk and plasma lutein concentrations were significantly correlated with dietary lutein intake $(r=0.86, P=0.0001$ and $r=0.94, P=0.0001$, respectively). Furthermore, there was a clear significant correlation between milk and plasma lutein concentrations ( $r=0 \cdot 87, P=0 \cdot 0001$; Fig. 1$)$.

In addition, breast milk lutein concentrations were significantly correlated with the number of portions of fruit $(2 \cdot 10$ (SD 1.06)) and vegetables $(1 \cdot 72(\mathrm{SD} 1 \cdot 20))$ consumed daily ( $r=0 \cdot 72, P=0 \cdot 01$ and $r=0 \cdot 68, P=0 \cdot 001$, respectively).

The fifteen subjects who ended the study provided another milk sample on day 30 postpartum (T1). On that occasion they also completed another FFQ.

At T0, estimated dietary lutein intake obtained from the FFQ of these fifteen subjects was 1209 (SD 157) $\mu \mathrm{g} / \mathrm{d}$; breast milk lutein and plasma lutein concentrations were $0.28(\mathrm{sD} 0.22$ ) and 0.67 (SD 0.42$) \mu \mathrm{mol} / \mathrm{l}$, respectively (Table 2). There were no significant differences among these variables compared with those of the whole sample.

At T1, estimated dietary lutein intake was 1258 (SD 102) $\mu \mathrm{g} / \mathrm{d}$, revealing no significant differences with either that estimated in the same sample ( $n 15 ; 1209$ (SD 157) $\mu \mathrm{g} / \mathrm{d}$, $P=0 \cdot 39$ ) or that estimated in the whole sample ( $n 21$; 1242 (sD 113) $\mu \mathrm{g} / \mathrm{d}, P=0 \cdot 27$ ) at T0.

Mature milk lutein concentration at T1 was $0 \cdot 11$ (SD $0 \cdot 05) \mu \mathrm{mol} / 1$ (Table 2). Plasma samples at T1 were not obtained. All the data were normally distributed.

Compared with the value reported at T0, mature milk lutein concentration decreased significantly over time $(P<0 \cdot 01)$, while there were no statistically significant changes in intake of this carotenoid over time. Breast milk lutein concentration at T1 maintained a fairly high correlation with estimated dietary lutein intake $(r=0.82$, $P=0 \cdot 0001$; Fig. 2).

\section{Discussion}

The present study aimed to estimate lutein concentration in human milk during early lactation and its relationship with dietary intake of this carotenoid.

Our population is small but there are many difficulties inherent to the enrolment of pregnant/postpartum women. On the other hand, we are confident that our results may add knowledge on research aimed at studying the protective role of these compounds in well-nourished pregnant women and their newborns.

The significant correlations observed at TO in the present study indicate that dietary intakes may provide valuable predictive information on maternal milk and
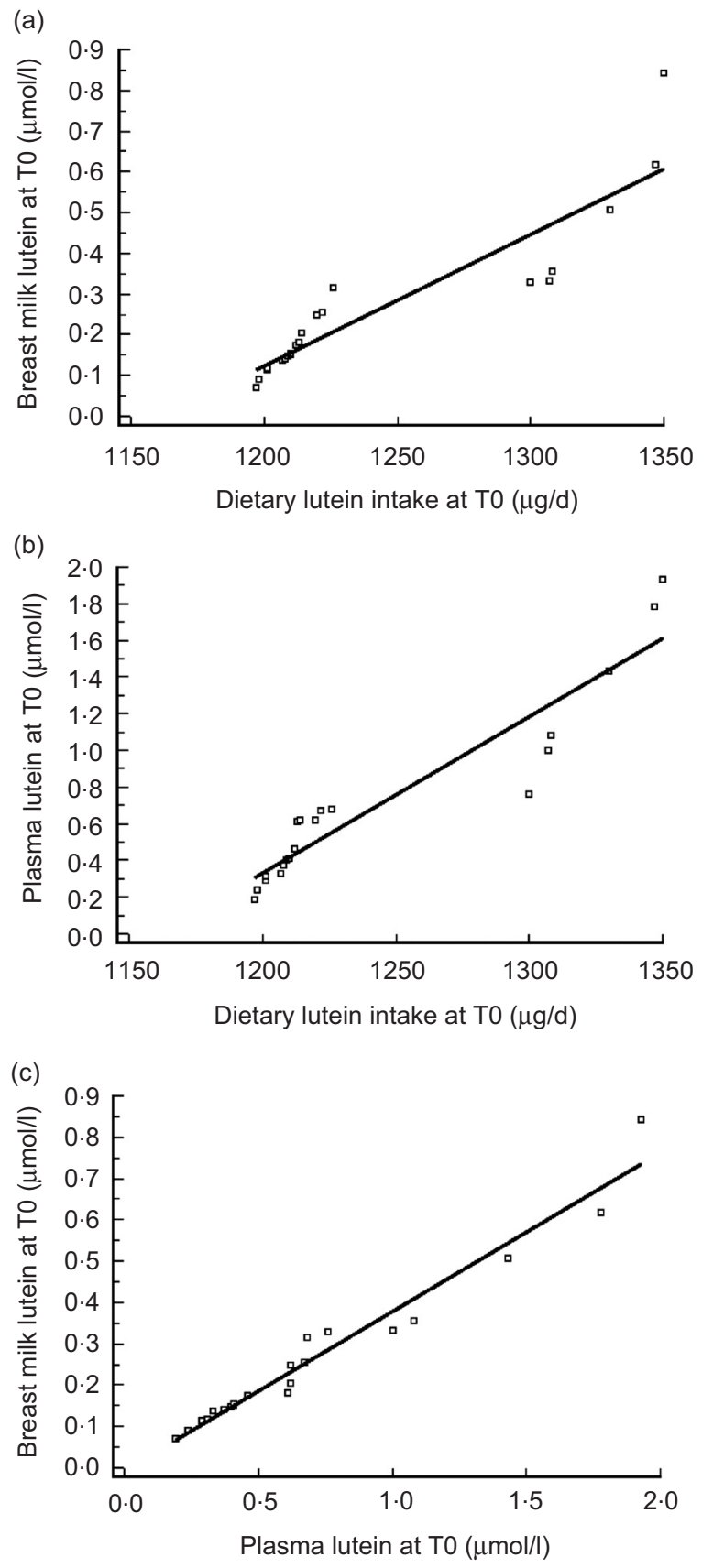

Fig. 1 Correlations between estimated dietary lutein intake and plasma and breast milk lutein concentrations at TO (day 3 postpartum) among lactating women ( $n 21)$ aged $24-42$ years, Pavia, Italy. All correlations were statistically significant: (a) $r=0.86, P=0.0001$; (b) $r=0.94, P=0.0001$; (c) $r=0.87$, $P=0.0001$

plasma lutein concentrations. In addition, correlations between breast milk lutein concentration and daily servings of fruit and vegetables appear to be sufficient as an indicator of dietary lutein intake, suggesting that eating selected fruit and vegetables regularly leads to a progressive increase in maternal milk lutein concentration.

Dietary lutein intake as well as milk and plasma lutein values measured in the present research are similar to 
Table 2 Lutein intake, mean plasma and breast milk lutein concentration in lactating women, Pavia, Italy

\begin{tabular}{|c|c|c|c|c|c|c|c|}
\hline \multirow[b]{2}{*}{ Time } & \multirow[b]{2}{*}{ No. of subjects } & \multicolumn{2}{|c|}{ Dietary lutein intake $(\mu \mathrm{g} / \mathrm{d})$} & \multicolumn{2}{|c|}{ Plasma lutein concentration $(\mu \mathrm{mol} / \mathrm{l})$} & \multicolumn{2}{|c|}{ Breast milk lutein concentration $(\mu \mathrm{mol} / \mathrm{l})$} \\
\hline & & Mean & SD & Mean & SD & Mean & SD \\
\hline TO & 15 & 1209 & 157 & 0.67 & $0 \cdot 42$ & $0 \cdot 28$ & $0 \cdot 22$ \\
\hline T1 & 15 & 1258 & 102 & - & - & $0 \cdot 11^{* *}$ & 0.05 \\
\hline
\end{tabular}

T0, day 3 postpartum; T1, day 30 postpartum.

Mean value was significantly different from that at T0 (Student's $t$ test): ${ }^{\star \star} P<0 \cdot 01$.

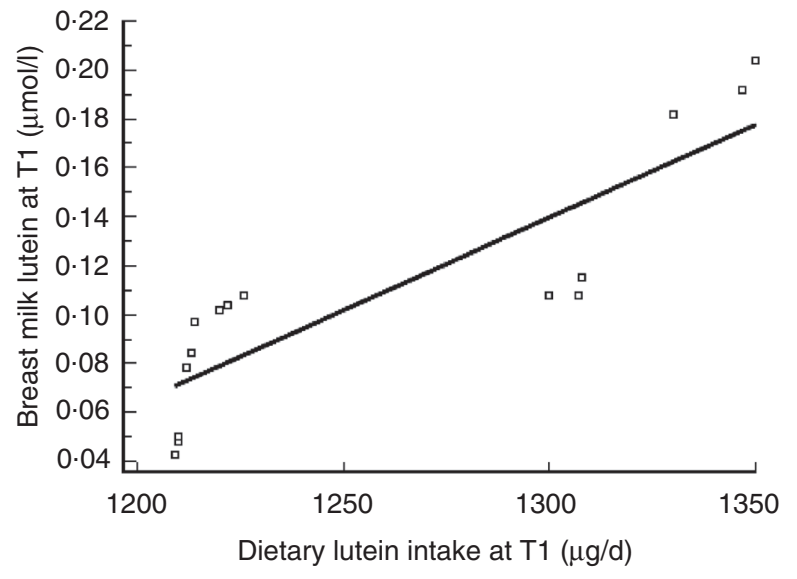

Fig. 2 Correlation between estimated dietary lutein intake and breast milk lutein concentration at T1 (day 30 postpartum) among lactating women $(n 15)$ aged $24-42$ years, Pavia, Italy. Correlation was statistically significant: $r=0.82, P=0.0001$

those of other studies, although authors report that both breast milk and plasma lutein concentrations show considerable inter-individual variability ${ }^{(25,26)}$.

We observed that dietary lutein intake was correlated both with breast milk $(r=0 \cdot 86, P=0 \cdot 0001)$ and plasma lutein concentrations $(r=0 \cdot 94, P=0 \cdot 0001)$, as already reported by other authors ${ }^{(11,27)}$. The strong correlation between dietary lutein intake and plasma lutein concentration is in agreement with our previous results ${ }^{(13)}$, as well as other data ${ }^{(28)}$. From further analyses a significant correlation also emerged between maternal milk and plasma lutein concentrations $(r=0 \cdot 87, P=0 \cdot 0001)$. A similarly high correlation has already been reported by other authors ${ }^{(29,30)}$, although weaker correlations have also been described in the literature ${ }^{(11)}$. Breast milk lutein concentration in our study was equal to approximately $40 \%$ of the plasma value, data that are supported by other studies $^{(12,31)}$.

The present study also sought to compare the difference in milk lutein concentration at 1 month postpartum, to observe changes in concentration of this carotenoid over time as well as changes in its relationship with dietary lutein intake.

Comparing lutein intakes assessed at T0 and T1, day 3 and 30 postpartum respectively, we determined that there were no changes in intake of this nutrient over time, assuming no significant variations in maternal diet composition. Daily lutein intake in our sample is similar to that obtained by other authors ${ }^{(13,18,32,33)}$.

According to the literature ${ }^{(12,31)}$, among lactating mothers in good health, breast milk carotenoid concentrations vary widely. Previous studies have identified women's dietary habits as the variable with the greatest influence on plasma carotenoid concentrations ${ }^{(34,35)}$; the same relationship seems to exist between dietary intakes and breast milk concentrations.

In our study, mature milk lutein concentration was significantly decreased $(P<0 \cdot 01)$ on day 30 postpartum, suggesting that lutein in breast milk appears to almost stabilize at 1 month postpartum. Our results are similar to those of many other studies, which have highlighted the fact that carotenoid concentrations (lutein, zeaxanthin, $\beta$-cryptoxanthin, lycopene, $\alpha$ - and $\beta$-carotene, retinol) in breast milk decrease over time, showing higher concentrations in colostrum compared with mature milk, enhancing however the importance of lutein compared with the others ${ }^{(11,22,25,36)}$. It has been reported that lutein represents approximately $25 \%$ of the milk carotenoid composition on day 3 postpartum, but approaches $50 \%$ on day 30 postpartum (mature milk) ${ }^{(11)}$. Other studies report these milk lutein concentration changes along with a decrease of plasma lutein concentration ${ }^{(37)}$. In our study, it would have been interesting therefore also to evaluate the mothers' plasma lutein concentrations at day 30 postpartum; the lack of such data highlights a limitation of our study.

Since dietary lutein intake did not change in our sample during the 1-month observation it may be suggested that the flow of lutein through the mammary gland could be altered in the postpartum period, implying that some event or set of events associated with parturition, initiation of lactation or both may affect lutein absorption, metabolism or both. These events might include postpartum normalization of lipoprotein concentration or changes in adiposity. It is difficult to speculate about the nature of these events on the basis of the limited data we collected.

The exact lutein secretion mechanism remains to be clarified, and may be different during colostrogenesis and the phases following lactogenesis. It could be linked to selective absorption of lipids through specific lipoprotein fractions, as indicated from the distribution pattern of 
carotenoids among these vehicles, or to particular mechanisms across membrane transporters and specific channels ${ }^{(25,29,38)}$.

\section{Conclusions}

Further research is needed to determine whether the observed postpartum decrease in milk lutein is simply normalization and to investigate the cause and potential role of breast milk lutein concentration changes during early lactation.

Even though milk lutein concentration decreases during early lactation, it remains significantly correlated to daily lutein intake. Therefore, while awaiting further research, dietary recommendations advising intake of fresh fruit and vegetables rich in lutein, throughout the whole duration of pregnancy and lactation, are extremely useful.

\section{Acknowledgements}

The present research was funded by the IRCCS Policlinico S. Matteo, Pavia, Italy. There are no financial or other contractual agreements that might cause conflicts of interest. Contributions of the authors are as follows: H.C. and G.T., conception and design of the study, generation and interpretation of data, revision of the manuscript; A.M.C., conception and design of the study, generation and collection of data; A.P., laboratory analysis; C.R., conception and design of the study. The authors state that: none of the material in the present manuscript is included in another manuscript nor has been or is currently under consideration for publication elsewhere; no portion of the manuscript has been published or posted on the Internet; ethical guidelines were followed by the authors in performing the study, which has been approved by the institutional human ethics committee; and each author has participated actively in the work as reported above and has given substantial contribution. Each author has read and approved the final submitted manuscript. The IRCCS Policlinico S. Matteo, Pavia, Italy is thanked for funding the research.

\section{References}

1. Landrum JT \& Bone RA (2001) Lutein, zeaxanthin, and the macular pigment. Arch Biochem Biophys 385, 28-40.

2. Krinsky NI (2002) Possible biologic mechanisms for a protective role of xanthophylls. J Nutr 132, Suppl., 540S-542S.

3. Landrum JT, Bone RA, Joa H, Kilburn MD, Moore LL \& Sprague KE (1997) A one year study of the macular pigment: the effect of 140 days of lutein supplement. Exp Eye Res 65, 57-62.

4. Bone RA, Landrum JT, Guerra LH \& Ruiz CA (2003) Lutein and zeaxanthin dietary supplements raise macular pigment density and serum concentrations of these carotenoids in humans. J Nutr 133, 992-998.
5. Conn PF, Schalch W \& Truscott TG (1991) The singlet oxygen and carotenoid interaction. J Photochem Photobiol $B$ 11, 41-47.

6. Junghans A, Sies H \& Stahl W (2001) Macular pigments lutein and zeaxanthin as blue light filters studied in liposomes. Arch Biochem Biophys 391, 160-164.

7. Jewell VC, Northrop-Clewes CA, Tubman R \& Thurnham DI (2001) Nutritional factors and visual function in premature infants. Proc Nutr Soc 60, 171-178.

8. Krinsky NI, Mayne ST \& Sies H (2004) Carotenoids in Health and Disease. New York: Marcel Dekker Ltd.

9. British Nutrition Foundation (1992) Unsaturated Fatty Acids: Nutritional and Physiological Significance. The Report of the British Nutrition Foundation's Task Force. London: Chapman \& Hall.

10. Hylander MA, Strobino DM, Pezzullo JC \& Dhanireddy R (2001) Association of human milk feedings with a reduction in retinopathy of prematurity among very low birthweight infants. J Perinatol 21, 356-362.

11. Gossage CP, Deyhim M, Yamini S, Douglass LW \& MoserVeillon PB (2002) Carotenoid composition of human milk during the first month postpartum and the response to $\beta$-carotene supplementation. Am J Clin Nutr 76, 193-197.

12. Canfield LM, Giuliano AR, Neilson EM, Yap HH, Graver EJ, Cui HA \& Blashill BM (1997) $\beta$-Carotene in breast milk and serum is increased after a single $\beta$-carotene dose. Am J Clin Nutr 66, 52-61.

13. Cena H, Roggi C \& Turconi G (2008) Development and validation of a brief food frequency questionnaire for dietary lutein and zeaxanthin intake assessment in Italian women. Eur J Nutr 47, 1-9.

14. Turrini A, Saba A, Perrone D, Cialfa E \& D'Amicis A (2001) Food consumption patterns in Italy: the INN-CA Study 1994-1996. Eur J Clin Nutr 55, 571-588.

15. Pasanisi P, Berrino F, Bellati C, Sieri S \& Krogh V (2002) Validity of the Italian EPIC questionnaire to assess past diet. IARC Sci Publ 156, 41-44.

16. Pala V, Berrino F, Vineis P, Palli D, Celentano E, Tumino R \& Krogh V (2002) How vegetables are eaten in Italy EPIC centres: still setting a good example? IARC Sci Publ 156, $119-121$.

17. Neuhouser ML, Rock CL, Eldridge AL, Kristal AR, Patterson RE, Cooper DA, Neumark-Sztainer D, Cheskin LJ \& Thornquist MD (2001) Serum concentrations of retinol, $\alpha$-tocopherol and the carotenoids are influenced by diet, race and obesity in a sample of healthy adolescents. J Nutr 131, 2184-2191.

18. Rock CL, Thornquist MD, Neuhouser ML, Kristal AR, Neumark-Sztainer D, Cooper DA, Patterson RE \& Cheskin LJ (2002) Diet and lifestyle correlates of lutein in the blood and diet. J Nutr 132, Suppl., 525S-530S.

19. Turconi G \& Roggi C (2007) Atlante fotografico alimentare: uno strumento per le indagini nutrizionali. Rome: Edizioni Mediche Scientifiche Internazionali.

20. Turconi G, Guarcello M, Gigli Berzolari F, Carolei A, Bazzano R \& Roggi C (2005) An evaluation of a colour food photography atlas as a tool for quantifying food portion sizes in epidemiological dietary surveys. Eur J Clin Nutr 59, 923-931.

21. Comstock GW, Alberg AJ \& Helzlsouer KJ (1993) Reported effects of long-term freezer storage on concentrations of retinol, $\beta$-carotene, and $\alpha$-tocopherol in serum or plasma summarized. Clin Chem 39, 1075-1078.

22. Jewell VC, Mayes CB, Tubman TR, Northrop-Clewes CA \& Thurnham DI (2004) A comparison of lutein and zeaxanthin concentrations in formula and human milk samples from Northern Ireland mothers. Eur J Clin Nutr 58, 90-97.

23. Barua AB (2001) Improved normal-phase and reversedphase gradient HPLC procedures for the analysis of 
retinoids and carotenoids in human serum, plant and animal tissues. $J$ Chromatogr $A$ 936, 71-82.

24. Holden JM, Eldridge AL, Beecher GR, Buzzard M, Bhagwat S, Davis CS, Douglass LW, Gebhardt S, Haytowitz D \& Schakel S (1999) Carotenoid content of US foods: an update of the data base. J Food Compost Anal 12, 169-196.

25. Macias C \& Schweigert FJ (2001) Changes in the concentration of carotenoids, vitamin A, $\alpha$-tocopherol and total lipids in human milk throughout early lactation. Ann Nutr Metab 45, 82-85.

26. Meneses F \& Trugo NMF (2005) Retinol, $\beta$-carotene, and lutein + zeaxanthin in the milk of Brazilian nursing women: associations with plasma concentrations and influences of maternal characteristics. Nutr Res 25, 443-451.

27. Canfield LM, Clandinin MT, Davies DP et al. (2003) Multinational study of major breast milk carotenoids of healthy mothers. Eur J Nutr 42, 133-141.

28. Bone RA, Landrum JT, Dixon Z, Chen Y \& Llerena CM (2000) Lutein and zeaxanthin in the eyes, serum and diet of human subjects. Exp Eye Res 71, 239-245.

29. Lietz G, Mulokozi G, Henry JC \& Tomkins AM (2006) Xanthophyll and hydrocarbon carotenoid patterns differ in plasma and breast milk of women supplemented with red palm oil during pregnancy and lactation. J Nutr 136, $1821-1827$

30. de Azeredo VB \& Trugo NM (2008) Retinol, carotenoids, and tocopherols in the milk of lactating adolescents and relationships with plasma concentrations. Nutrition $\mathbf{2 4}$, 133-139.

31. Canfield LM, Giuliano AR, Neilson EM, Blashil BM, Graver EJ \& Yap HH (1998) Kinetics of the response of milk and serum $\beta$-carotene to daily $\beta$-carotene supplementation in healthy, lactating women. Am J Clin Nutr 67, 276-283.

32. Curran-Celentano J, Hammond BR Jr, Ciulla TA, Cooper DA, Pratt LM \& Danis RB (2001) Relation between dietary intake, serum concentrations, and retinal concentrations of lutein and zeaxanthin in adults in a Midwest population. Am J Clin Nutr 74, 796-802.

33. Hammond BR Jr, Ciulla TA \& Snodderly DM (2002) Macular pigment density is reduced in obese subjects. Invest Ophthalmol Vis Sci 43, 47-50.

34. Broekmans WM, Klöpping-Ketelaars IA, Schuurman CR, Verhagen $H$, van den Berg H, Kok FJ \& van Poppel G (2000) Fruits and vegetables increase plasma carotenoids and vitamins and decrease homocysteine in humans. J Nutr 130, 1578-1583.

35. Tucker KL, Chen H, Vogel S, Wilson PW, Schaefer EJ \& Lammi Keefe CJ (2002) Carotenoid intakes, assessed by dietary questionnaire, are associated with plasma carotenoid concentrations in an elderly population. J Nutr 129, 438-445.

36. Sommerburg OG, Siems WG, Hurst JS, Lewis JW, Kliger DS \& van Kuijk FJ (1999) Lutein and zeaxanthin are associated with photoreceptors in the human retina. Curr Eye Res 19, 491-495.

37. Gossage C, Deyhim M, Moser-Veillon PB, Douglas LW \& Kramer TR (2000) Effect of $\beta$-carotene supplementation and lactation on carotenoid metabolism and mitogenic $\mathrm{T}$ lymphocyte proliferation. Am J Clin Nutr 71, 950-955.

38. Schweigert FJ, Bathe K, Chen F, Büscher U \& Dudenhausen JW (2004) Effect of the stage of lactation in humans on carotenoid levels in milk, blood plasma and plasma lipoprotein fractions. Eur J Nutr 43, 39-44. 